\title{
Bulk, surface and corner free energy series for the chromatic polynomial on the square and triangular lattices
}

\author{
Jesper Lykke Jacobsen \\ LPTENS, École Normale Supérieure, 24 rue Lhomond, 75231 Paris, France \\ Université Pierre et Marie Curie, 4 place Jussieu, 75252 Paris, France \\ E-mail: jesper.jacobsen@ens.fr
}

\begin{abstract}
.
We present an efficient algorithm for computing the partition function of the $q$ colouring problem (chromatic polynomial) on regular two-dimensional lattice strips. Our construction involves writing the transfer matrix as a product of sparse matrices, each of dimension $\sim 3^{m}$, where $m$ is the number of lattice spacings across the strip. As a specific application, we obtain the large- $q$ series of the bulk, surface and corner free energies of the chromatic polynomial. This extends the existing series for the square lattice by 32 terms, to order $q^{-79}$. On the triangular lattice, we verify Baxter's analytical expression for the bulk free energy (to order $q^{-40}$ ), and we are able to conjecture exact product formulae for the surface and corner free energies.
\end{abstract}

\section{Introduction}

The $q$-colouring problem consists in assigning to each of the vertices of a graph $G$ any one of $q$ different colours, in such a way that adjacent vertices carry different colours. The number of possible colourings (possibly zero) is known as the chromatic polynomial $P_{G}(q)$ of the graph. Although this definition supposes that $q$ is a positive integer, it is not hard to show [1, 2] that $P_{G}(q)$ is indeed a polynomial in $q$, and so the number of colours can be regarded as a formal variable.

The history of the $q$-colouring problem is long and interesting, and we refer the reader to [3] for an extensive list of references. The case where $G$ is a planar graph has attracted particular interest. A well-known result is then the four-colouring theorem [4] which states that $P_{G}(4)>0$ for any planar $G$. Other results exploit the extension of $P_{G}(q)$ to non-integer values of $q$. One interesting question is whether there exists, in the planar case, some $q_{\mathrm{c}}$ so that $P_{G}(q)>0$ for all $q \in\left[q_{\mathrm{c}}, \infty\right)$. This statement has been established as a theorem [5] for $q_{\mathrm{c}}=5$, and the so-called Birkhoff-Lewis conjecture [5] that this extends to $q_{\mathrm{c}}=4$ is widely believed to be true.

Apart from these general results, the case where $G$ is a regular planar latticetypically square or triangular - has also been intensively studied. In particular, a long 
series of papers $[6,[3,7,6,9,10,11,12]$ is devoted to the study of the location and properties of chromatic zeroes, $P_{G}(q)=0$, in the complex $q$-plane for a variety of boundary conditions. Some of the mechanisms responsible for the generation of real chromatic zeroes in the region $q \in[0,4]$ have even been understood analytically [13, 14]. One should also mention that there exists a family of planar graphs with real chromatic roots converging to $q=4$ from below [15].

An important technical ingredient in the studies of chromatic zeroes is the possibility to build up $P_{G}(q)$, for regular lattice strips of a given width $m$, by means of a transfer matrix $T$. In order to elucidate the amazingly intricate behaviour of the chromatic polynomial in the thermodynamical limit, it is clearly desirable to be able to access as large $m$ as possible in these computations.

In the present paper we show how to improve the constructions of [16, 3, 17, 8, 11] by writing $T$ as a product of sparse matrices, each of dimension $\sim 3^{m}$. This is implemented in an efficient way, that enables us to compute exactly $P_{G}(q)$ for rectangles as large as $20 \times 21$. Using the finite-lattice method [17] this results in series expansions - in powers of $1 / q$ - of the bulk, surface and corner free energies of the chromatic polynomial. To give an idea of the improvement on existing results, the bulk free energy series on the square lattice was taken to order $q^{-36}$ in [18], and very recently extended to order $q^{-47}$ in [11. We here add a further 32 terms to this latter series, taking it to order $q^{-79}$. The surface and corner free energies are similarly obtained to orders $q^{-79}$ and $q^{-78}$.

The case of the triangular lattice - which does not appear to have been studied previously in terms of series expansions of the free energy - turns out to be particularly interesting. Baxter [19, 6] has shown that for this lattice the chromatic polynomial possesses an integrable structure. This permitted him to derive an analytical expression for the bulk free energy, in the form of an infinite product formula, valid in the region $q \in(-\infty, 0] \cup[4, \infty)$. We obtain in this case the bulk, surface and corner free energies to order $q^{-40}$. Our results validate Baxter's result for the bulk free energy, and permit us to conjecture also the exact infinite product formulae for the surface and corner free energies.

\section{Chromatic polynomial}

We first recall how the chromatic polynomial appears as a special case of the partition function of the $q$-state Potts model. Given a finite undirected graph $G=(V, E)$ with vertex set $V$ and edge set $E$, the Hamiltonian of the Potts model is given by

$$
H=-J \sum_{(i j) \in E} \delta_{\sigma_{i}, \sigma_{j}}
$$

where $J$ is the coupling constant, and a spin $\sigma_{i} \in\{1,2, \ldots, q\}$ is defined at each vertex $i \in V$. The Kronecker delta function $\delta_{\sigma_{i}, \sigma_{j}}$ equals 1 if $\sigma_{i}=\sigma_{j}$, and 0 otherwise.

Setting $v=\mathrm{e}^{J}-1$, the partition function can be rewritten as

$$
Z_{G}(q, v)=\sum_{\{\sigma\}} \prod_{(i j) \in E}\left[1+v \delta_{\sigma_{i}, \sigma_{j}}\right]
$$


Free energy series for the chromatic polynomial

$$
=\sum_{A \subseteq E} q^{k(A)} v^{|A|}
$$

where $k(A)$ is the number of connected components in the subgraph $G_{A}=(V, A)$, and $|A|$ is the number of elements in the edge subset $A \subseteq E$. In the rewriting (3)-known as the Fortuin-Kasteleyn (FK) representation [20] $-q$ does no longer need to be an integer, and in particular can be used as an expansion parameter. For $q \rightarrow \infty$, only the term with $A=\emptyset$-whence $k(A)=|V|$-contributes, and thus one can develop $Z_{G}(q, v)$ in powers of $1 / q$. The chromatic polynomial appears as the special case $v=-1$,

$$
P_{G}(q)=Z_{G}(q,-1),
$$

as is evident from (2).

\section{Transfer matrix formalism}

Thanks to the FK representation (3), the partition function for a lattice strip of width $m$ can be built using a transfer matrix $T$ of dimension independent of $q$ [16, 3]. In this section we briefly review the necessary formalism, with the minor modification that we rewrite things in terms of $q^{-1}$ rather than $q$, since this is more convenient for our series expansion purposes. We then introduce several improvements:

(i) A factorisation of $T$ into sparse matrices that requires only the use of non-nearestneighbour and almost non-nearest-neighbour partitions.

(ii) An efficient labelling of non-nearest-neighbour partitions by natural numbers.

(iii) The use of modular arithmetic instead of arbitrary-precision integer arithmetic.

The combination of these ingredients will yield an efficient algorithm that allows, in particular, to add further terms to the series expansions of [18, 11.

Let us start by noting that, in view of (3),$T$ can be taken to act on states which are partitions of the vertex set $\{1,2, \ldots, m\}$ corresponding to a single horizontal layer of spins. The role of $T$ is to propagate the system one lattice spacing upwards in the vertical direction. The states describe how the connected components of $G_{\underline{A}}=(V, \underline{A})$ partition the $m$ vertices of a layer, where $\underline{A}$ is the intersection of $A$ with the set of edges which are below the layer under consideration. For instance, when $m=3$, the five possible states are the partitions

$$
(1)(2)(3), \quad(12)(3), \quad(13)(2), \quad(1)(23), \quad(123) .
$$

We shall see shortly how the information contained in these states permits us to account for the non-locality of the Boltzmann weight $q^{k(A)}$.

Our objective is to write the chromatic polynomial on a rectangular piece, of width $m$ and height $n$, of the square or triangular lattice in the form

$$
P_{G}(q)=\left\langle e_{0}\left|T_{\text {last }} T^{n-1}\right| e_{0}\right\rangle
$$

where the basis state $\left|e_{0}\right\rangle$ corresponds to the all-singleton partition $(1)(2) \cdots(m)$. Each transfer matrix $T$ adds one layer to the lattice, consisting of $m-1$ horizontal edges and 
$m$ vertical edges - and for the triangular lattice also $m-1$ diagonal edges. A special matrix $T_{\text {last }}$ will be required to deal with the last layer of $m-1$ horizontal edges. The notation $\left\langle e_{0}\right|$ means projection on the all-singleton state, and is not meant to imply the definition of a scalar product on the space of partitions (although such a scalar product can indeed be defined).

Let us observe that it would be quite wasteful to construct the matrix $T$ explicitly. Not only would this require memory resources of the order of $(\operatorname{dim} T)^{2}$ for its storage, but computing the product $T|v\rangle$, where $|v\rangle$ represents an arbitrary linear combination of all possible basis states, would take a time proportional to $(\operatorname{dim} T)^{2}$. If, on the other hand, we write $T$ as a product of matrices each corresponding to the addition of a single edge, these matrices will turn out to be sparse (with only one or two non-zero entries per column), and so both time and memory requirements are linear in the dimension of these matrices. In order to proceed, we first study these single-edge matrices in some detail.

\subsection{Single-edge transfer matrices}

Since we are going to construct a series expansion in powers of $1 / q$, it will be useful to pull out a trivial factor in (3) and study instead

$$
\tilde{Z}_{G}(q, v)=q^{-|V|} Z_{G}(q, v)=\sum_{A \subseteq E} q^{k(A)-|V|} v^{|A|} .
$$

The lowest-order term, corresponding to $A=\emptyset$, is then 1 . It is useful to think of the factor $q^{k(A)-|V|}$ in the following way: whenever the addition of an edge to the set $A$ joins up two connected component the result is a factor of $q^{-1}$.

The single-edge transfer matrix that adds a horizontal edge between vertices $i$ and $i+1$ can now be written

$$
\mathrm{H}_{i, i+1}=1+v \mathrm{~J}_{i, i+1},
$$

where the first (resp. second) term represents the possibility of that edge being absent from (resp. present in) the subset $A$. The join operator $J_{i, i+1}$ acts on a partition of $\{1,2, \ldots, m\}$ as the identity if $i$ and $i+1$ belong to the same block. If they belong to two different blocks, the action of $\mathrm{J}_{i, i+1}$ is to join up the blocks and attribute a factor $q^{-1}$.

We now make a remark that will turn out to be very important in what follows. For the case of the chromatic polynomial (i.e., $v=-1$ ) $\mathrm{H}_{i, i+1}$ is idempotent. It projects out states in which $i$ and $i+1$ belong to the same block. It turns out useful to introduce the normalised projection operator $\mathrm{P}_{i, i+1}$ which acts on a partition as the identity if $i$ and $i+1$ belong to different blocks, and as zero if $i$ and $i+1$ are in the same block. We have then

$$
\mathrm{H}_{i, i+1} \mathrm{P}_{i, i+1}=\mathrm{H}_{i, i+1}=\left(\mathrm{H}_{i, i+1}\right)^{2} .
$$

The transfer matrix corresponding to a vertical edge between vertices $i$ and $i^{\prime}$ can be thought of as the following sequence of operations: 
(i) Insert $i^{\prime}$ into the partition as a singleton.

(ii) Apply $\mathrm{H}_{i, i^{\prime}}$.

(iii) Eliminate $i$ from the partition.

(iv) Relabel $i^{\prime}$ as $i$.

Alternatively, the vertical edge as can be represented as the operator

$$
\mathrm{V}_{i}=v q^{-1}+\mathrm{D}_{i},
$$

where the first (resp. second) term represents the situation where that edge is present in (resp. absent from) the subset $A$. The detach operator $\mathrm{D}_{i}$ acts by transforming $i$ into a singleton. The advantage of the rewriting is that the relabelling $i^{\prime} \rightarrow i$ is done automatically, but one should keep in mind that $i$ does not represent the same vertex on the lattice before and after the action of $\mathrm{V}_{i}$.

Note that although the vertical-edge transfer matrix $\mathrm{V}_{i}$ is related to $\mathrm{H}_{i, i^{\prime}}$, it is not a projector in the chromatic case $v=-1$.

We next show how to combine the observation that $\mathrm{H}_{i, i+1}$ is a projector with the sparse matrix decomposition of $T$ in order to achieve a very efficient transfer matrix formalism. Although this can be done for any regular lattice, we shall discuss only the cases of the square and triangular lattices.

\subsection{Square lattice}

One can use the operators $\mathrm{H}_{i, i+1}$ and $\mathrm{V}_{i}$ to build up the lattice, by writing $T$ as a suitable product of such operators. An obvious construction is to first lay down all the horizontal edges of a layer, and the add all vertical edges that connect to the next layer:

$$
T=\left(\prod_{i=1}^{m} \mathrm{\vee}_{i}\right)\left(\prod_{i=1}^{m-1} \mathrm{H}_{i, i+1}\right) \quad(\text { any } v) .
$$

Since the next transfer matrix in the product (6) will first lay down a row of horizontal edges, we may use (9) and write instead, for $v=-1$,

$$
T=\left(\prod_{i=1}^{m-1} \mathrm{P}_{i, i+1}\right)\left(\prod_{i=1}^{m} \mathrm{~V}_{i}\right)\left(\prod_{i=1}^{m-1} \mathrm{H}_{i, i+1}\right) \quad(v=-1 \text { only }) .
$$

Although the construction (12) takes advantage of the sparse matrix decomposition, it is not maximally efficient for reasons that we now point out.

Consider first the case of generic $v$. Successive applications of $T$ to $\left|e_{0}\right\rangle$, as in (6), will generate a number of basis states corresponding to all possible partitions of $\{1,2, \ldots, m\}$ that are consistent with planarity. These are known in the literature as non-crossing partitions, and their number is given by the Catalan number

$$
C_{m}=\frac{(2 m) !}{m !(m+1) !}=1,2,5,14,42,132, \ldots,
$$

which grows asymptotically as $C_{m} \sim m^{-3 / 2} 4^{m}$ for $m \gg 1$. 
For $v=-1$ we can use (12), and so once a layer is completed the number of basis states equals the number of non-crossing non-nearest-neighbour partitions of $\{1,2, \ldots, m\}$. Their number is given [3] by the Motzkin number $M_{m-1}$, where

$$
M_{k}=\sum_{j=0}^{\lfloor k / 2\rfloor}\left(\begin{array}{c}
k \\
2 j
\end{array}\right) C_{j}=1,1,2,4,9,21, \ldots .
$$

For instance, when $m=4$, the four possible such partitions read

$$
(1)(2)(3)(4), \quad(1)(24)(3), \quad(13)(2)(4), \quad(14)(2)(3) .
$$

The asymptotic behaviour is now $M_{k} \sim k^{-3 / 2} 3^{k}$ for $k \gg 1$. This looks like a considerable improvement. However, the intermediate stages that appear when building up the product (12) still require all nearest-neighbour partitions, and so the time necessary for a complete multiplication with $T$ still grows like $4^{m}$.

Fortunately, we can write the sparse matrix decomposition of $T$ in a way which (essentially) only makes use of non-crossing non-nearest-neighbour partitions. This is accomplished by utilising the property (9) maximally. Indeed we can write, for $v=-1$,

$$
T=T_{m} T_{m-1} \ldots T_{3} T_{2} T_{1}
$$

with

$$
\begin{aligned}
T_{i} & =\left(\prod_{\substack{j=1 \\
j \neq i}}^{m-1} \mathrm{P}_{j, j+1}\right) \mathrm{V}_{i} \mathrm{H}_{i, i+1} \quad \text { for } i=1,2, \ldots, m-1 \\
T_{m} & =\left(\prod_{j=1}^{m-1} \mathrm{P}_{j, j+1}\right) \mathrm{V}_{m} .
\end{aligned}
$$

After the multiplication by $T_{i}$, the non-nearest-neighbour constraint is now in action everywhere, except possibly between sites $i$ and $i+1$. The number of partitions where $i$ and $i+1$ do belong to the same block is $M_{m-2}$, since for such partitions the contraction of $i$ and $i+1$ into a single point clearly leads to a non-crossing non-nearest-neighbour partition on $m-1$ points.

The sparse-matrix factorisation (15)-(16) is a central result of this paper. It implies that, for the square lattice, the number of basis states needed to compute the chromatic polynomial using (6) and (15) is $M_{m-1}$ when a layer has just been completed, and $M_{m-1}+M_{m-2}$ in the intermediate stages. Both numbers grow like $3^{m}$.

It remains to describe how the matrix $T_{\text {last }}$ lays down the last layer of horizontal edges. As before this is done one edge at a time

$$
T_{\text {last }}=T_{m}^{\text {last }} T_{m-1}^{\text {last }} \ldots T_{3}^{\text {last }} T_{2}^{\text {last }} T_{1}^{\text {last }}
$$

In this product, each factor adds a horizontal bond between $i$ and $i+1$. Having done that, vertex $i$ and those to the left of it are of no use any longer, so we might as well detach them as singletons. This leads to

$$
T_{i}^{\mathrm{last}}=\left(\prod_{j=i+1}^{m-1} \mathrm{P}_{j, j+1}\right) \mathrm{D}_{i} \mathrm{H}_{i, i+1} \quad \text { for } i=1,2, \ldots, m .
$$


Note that after the action of $T_{\text {last }}$, only the all-singleton state $\left|e_{0}\right\rangle$ remains. The chromatic polynomial of $G$ appears as the coefficient of this state, leading finally to (6).

\subsection{Triangular lattice}

The triangular lattice can be drawn as a square lattice with diagonals. These diagonals can be constructed, within the transfer matrix approach, by applying the operators $\mathrm{H}_{i, i+1}$ just after site $i$ has been propagated from one layer to the next by means of $\mathrm{V}_{i}$, but before $\mathrm{V}_{i+1}$ has been employed.

We have again the factorisation (15), but now with

$$
\begin{aligned}
T_{1} & =\left(\prod_{j=1}^{m-1} \mathrm{P}_{j, j+1}\right) \mathrm{V}_{1} \mathrm{H}_{1,2} \\
T_{i} & =\left(\prod_{j=1}^{m-1} \mathrm{P}_{j, j+1}\right) \mathrm{V}_{i} \mathrm{H}_{i-1, i} \mathrm{H}_{i, i+1} \quad \text { for } i=2,3, \ldots, m-1 \\
T_{m} & =\left(\prod_{j=1}^{m-1} \mathrm{P}_{j, j+1}\right) \mathrm{V}_{m} \mathrm{H}_{m-1, m} .
\end{aligned}
$$

Note that, due to the existence of the diagonal edges, the leftmost factor in each expression is the product over all nearest-neighbour projectors. Therefore, the number of basis states required in any intermediate stage of the construction is $M_{m-1}$.

The expressions (17)-(18) for $T_{\text {last }}$ are identical to those for the square lattice.

\subsection{Ordering the states}

At this point, one would be ready to write a transfer matrix for the chromatic polynomial using only non-crossing non-nearest-neighbour (NCNNN) partitions - up to a small subtlety for the square lattice - as intermediate states, by storing the partitions encountered using standard hashing techniques.

One can however reduce further - albeit only by a constant factor - time and memory requirements by establishing a bijection between the natural numbers $\left\{1,2, \ldots, M_{m-1}\right\}$ and the set of $M_{m-1}$ NCNNN partitions. Using this, the vectors appearing in the computation of (6) can be realised simply as standard arrays, indexed by the natural numbers, and each basis state can be mapped back and forth between its representation as a natural number and as a partition on which the operators $\mathrm{H}_{i, i+1}, \mathrm{~V}_{i}$ and $\mathrm{P}_{i, i+1}$ can be made to act. This idea was previously implemented in the context of the planar Potts model (i.e., for non-crossing partitions) by Blöte and Nightingale [16], and we extend it here by adding the non-nearest-neighbour constraint.

An important step in establishing the bijection is to produce an ordering of the NCNNN partitions for a given $m$. To this end, consider the first point in the partition. Two situations may arise: 
(i) If point 1 is a singleton, the restriction of the partition to the points $\{2,3, \ldots, m\}$ is again NCNNN.

(ii) If point 1 is not a singleton, let $k \in\{0,1, \ldots, m-3\}$ be the smallest integer so that point $3+k$ belongs to the same block as 1 . Then the restriction of the partition to points $\{2,3, \ldots, 2+k\}$ and the restriction to points $\{3+k, 4+k, \ldots, m\}$ are two independent NCNNN partitions of respectively $k+1$ and $m-k-2$ points.

This argument proves in particular the recursion formula (with $p=m-1$ )

$$
M_{p}=M_{p-1}+\sum_{k=0}^{p-2} M_{k} M_{p-k-2}
$$

with initial values $M_{0}=M_{1}=1$.

Suppose now, as an induction hypotheses, that we have defined an ordering of NCNNN partitions of fewer than $m$ points. The ordering of NCNNN partitions of 1 or 2 points is trivial, since in these cases there is only one such partition. We can then order NCNNN partitions of $m$ points as follows:

(i) Place first the partitions in which point 1 is a singleton. The ordering of these partitions follows inductively from the ordering of the partitions of points $\{2,3, \ldots, m\}$.

(ii) Place next the partitions in which point 1 is not a singleton, in the order of increasing values of the integer $k$ defined above. The ordering of the partitions with a fixed value of $k$ follows inductively from the ordering of the partitions of points $\{2,3, \ldots, 2+k\}$ and $\{3+k, 4+k, \ldots, m\}$, using the former (resp. latter) as the most (resp. least) significant 'bit'.

For instance, the ordering for $m=5$ points reads:
$(1)(2)(3)(4)(5)$,
$(1)(2)(35)(4)$,
$(1)(24)(3)(5)$
$(1)(25)(3)(4)$,
$(13)(2)(4)(5)$,
$(135)(2)(4)$
$(14)(2)(3)(5)$,
$(15)(2)(3)(4)$,
$(15)(24)(3)$.

The mapping from integers to partitions can now be constructed by inferring the value of $k$ from (20) and proceeding recursively. The inverse mapping is constructed similarly by obtaining $k$ explicitly from the partition.

Finally, for the square lattice, we need a bijection between the integers $\left\{1,2, \ldots, M_{m-1}+M_{m-2}\right\}$ and partitions in which points $i$ and $i+1$ are allowed to be in the same block. Again this follows from our ability to order such partitions. To this end, we place first the $M_{m-1}$ partitions in which $i$ and $i+1$ are not in the same block, and next the $M_{m-2}$ partitions in which $i$ and $i+1$ are in the same block. The remaining ingredients of the bijection can be taken over directly from the NCNNN case.

\subsection{Implementational details}

We wish to compute the chromatic polynomial, normalised as in (7),

$$
\tilde{P}_{G}(q)=q^{-|V|} Z_{G}(q,-1),
$$


where $G$ is an $m \times n$ rectangular section of the square or triangular lattice (the latter being considered as a square lattice with added diagonals). The result is a polynomial of degree $|V|-1$ in the variable $q^{-1}$ with constant term 1 . To obtain this by the transfer matrix method, each NCNNN state must have a coefficient (weight) which is a polynomial of degree $d=|V|-1$ with integer coefficients. These coefficients can be stored quite simply as an integer array of length $d+1$.

Since the goal is to produce a series expansion in powers of $q^{-1}$ of the various free energies, there is actually no need to compute the whole polynomial $\tilde{P}_{G}(q)$. We shall see below that one knows in advance to which degree $d_{\max }$ the expansion can be taken. It suffices therefore to truncate all polynomials intervening in the coefficients to that order. Such a truncation leads to non-negligible savings of memory resources.

A practical problem arises because the integer coefficients rapidly become very large. This is effectively handled by using modular arithmetic, i.e., by computing

the coefficients $\tilde{P}_{G}(q)$ modulo various primes $p_{i}$. Since the transfer process only uses additions and subtractions, it is convenient to use 32-bit unsigned integers in the computations and to choose $p_{i}<2^{31}$ in order to avoid overflow before each modulo operation can be carried out. Using the Chinese remainder theorem, the partial results $\tilde{P}_{G}(q) \bmod p_{i}$ can be combined to give $\tilde{P}_{G}(q) \bmod \prod_{i} p_{i}$. Once the latter stabilises upon using further primes we know that we have arrived at the complete result $\tilde{P}_{G}(q)$, with no modulo. The results reported below made necessary the use of up to 16 different primes.

\section{Results}

We first review briefly how the series expansion for the free energies can be obtained from the chromatic polynomials on finite $m \times n$ rectangles $G_{m, n}$ by use of the finitelattice method [17]. The goal is to obtain a development for the free energy per vertex in the form

$$
\begin{aligned}
f_{m, n}(q) & \equiv \frac{\log P_{G}(q)}{m n} \\
& =\left[\log q+\sum_{k=0}^{\infty} \frac{a_{k}}{q^{k}}\right]+\left[\sum_{k=0}^{\infty} \frac{b_{k}}{q^{k}}\right]\left(\frac{1}{m}+\frac{1}{n}\right)+\left[\sum_{k=0}^{\infty} \frac{c_{k}}{q^{k}}\right] \frac{1}{m n},
\end{aligned}
$$

exactly valid, order by order in $q^{-1}$, for all $m$ and $n$ sufficiently large. The quantities within square brackets are referred to as the bulk, surface and corner free energies, $f_{\mathrm{b}}(q)$, $f_{\mathrm{s}}(q)$ and $f_{\mathrm{c}}(q)$.

\subsection{Finite-lattice method}

We discuss first the case of the square lattice. The free energies introduced above are given by the finite-lattice method [17] as

$$
f_{\mathrm{b}}(q)=\log q+\sum_{(r, s) \in B(k)} \alpha_{k}(r, s) \log \tilde{P}_{G(r, s)}(z)+O\left(z^{2 k-3}\right),
$$


Free energy series for the chromatic polynomial

$$
\begin{aligned}
& f_{\mathrm{s}}(q)=\sum_{(r, s) \in B(k)} \beta_{k}(r, s) \log \tilde{P}_{G(r, s)}(z)+O\left(z^{2 k-3}\right), \\
& f_{\mathrm{c}}(q)=\sum_{(r, s) \in B(k)} \gamma_{k}(r, s) \log \tilde{P}_{G(r, s)}(z)+O\left(z^{2 k-3}\right),
\end{aligned}
$$

where the set $B(k)$ are $r \times s$ rectangles $G(r, s)$ such that $r \leq s$ and $r+s \leq k$. For convenience - and to facilitate the comparison with previous work [18, 11] - the expansion variable $q^{-1}$ has here been changed to

$$
z=\frac{1}{q-1} \text {. }
$$

The error term $O\left(z^{2 k-3}\right)$ in (23)-(25) is due to certain classes of convex polygons [17] which do not fit into any of the rectangles in $B(k)$.

The finite-lattice weights for $f_{\mathrm{b}}$ are given by [17]

$$
\alpha_{k}(r, s)=\left(2-\delta_{r, s}\right)\left(\delta_{r+s, k}-3 \delta_{r+s, k-1}+3 \delta_{r+s, k-2}-\delta_{r+s, k-3}\right)
$$

while those for $f_{\mathrm{s}}$ and $f_{\mathrm{c}}$ read [21]

$$
\begin{aligned}
\beta_{k}(r, s)= & \left(2-\delta_{r, s}\right)\left(\frac{2-r-s}{2} \delta_{r+s, k}+\frac{3(r+s)-2}{2} \delta_{r+s, k-1}-\right. \\
& \left.\frac{3(r+s)+2}{2} \delta_{r+s, k-2}+\frac{r+s-2}{2} \delta_{r+s, k-3}\right) \\
\gamma_{k}(r, s)= & \left(2-\delta_{r, s}\right)\left((r-1)(s-1) \delta_{r+s, k}+(1+r+s-3 r s) \delta_{r+s, k-1}+\right. \\
& \left.(3 r s+r+s-1) \delta_{r+s, k-2}-(r+1)(s+1) \delta_{r+s, k-3}\right) .
\end{aligned}
$$

\subsection{Square lattice series}

Using the transfer matrix formalism we can compute $\tilde{P}_{G(r, s)}$ for lattice strips of width $r \leq$ $m_{\max }$. Without resorting to super computer facilities or massive parallel computations, we have found that $m_{\max }=20$ was feasible on a standard workstation. This corresponds to a maximum dimension of the transfer matrices of $M_{19}+M_{18}=24735666$ on the square lattice. Setting $k=41$ above, this gives then the free energies $f_{\mathrm{b}}, f_{\mathrm{s}}$ and $f_{\mathrm{c}}$ correctly to order $z^{78}$. For the case of $f_{\mathrm{b}}$ and $f_{\mathrm{s}}$ an extra term can be obtained by adding manually the term $\mu_{2 m_{\max }} z^{4 m_{\max }-1}$ to $f_{\mathrm{b}}[18]$, and the term $-m_{\max } \mu_{2 m_{\max }} z^{4 m_{\max }-1}$ to $f_{\mathrm{s}}$ [11], where [18]

$$
\sum_{p=4}^{\infty} \mu_{p} x^{2 p}=x^{8} \frac{2-2 x^{2}-x^{2} \sqrt{1-4 x^{2}}}{\left(1-4 x^{2}\right)\left(2+x^{2}\right)}+x^{12} \frac{3-4 x^{2}-4 \sqrt{1-4 x^{2}}}{\left(1-4 x^{2}\right)^{2}} .
$$

We thus obtain the series to order $z^{79}$ in these cases.

The results for all three series are given in Table 1. They extend those of [1] by 32 new terms.

It is possible to analyse $₫$ the extended series using the method of differential approximants. The goal is to obtain the locations $z_{\ell}$ and exponents $\lambda_{\ell}$ of the singularities $\ddagger$ We are grateful to J. Salas for providing the analysis mentioned in this paragraph. 
Free energy series for the chromatic polynomial

\begin{tabular}{|c|c|c|c|}
\hline$k$ & $\alpha_{k}$ & $\beta_{k}$ & $\gamma_{k}$ \\
\hline 0 & 1 & 1 & 1 \\
\hline 1 & 0 & 1 & 0 \\
\hline 2 & 0 & 0 & 0 \\
\hline 3 & 1 & -1 & 1 \\
\hline 4 & 0 & -1 & 0 \\
\hline 5 & 0 & 0 & 0 \\
\hline 6 & 0 & 1 & 0 \\
\hline 7 & 1 & -1 & 4 \\
\hline 8 & 3 & -8 & 12 \\
\hline 9 & 4 & -16 & 20 \\
\hline 10 & 3 & -16 & 28 \\
\hline 11 & 3 & -12 & 67 \\
\hline 12 & 11 & -41 & 208 \\
\hline 13 & 24 & -138 & 484 \\
\hline 14 & 8 & -210 & 753 \\
\hline 15 & -91 & 47 & 750 \\
\hline 16 & -261 & 849 & 679 \\
\hline 17 & -290 & 1471 & 2320 \\
\hline 18 & 254 & -493 & 10020 \\
\hline 19 & 1671 & -8052 & 30548 \\
\hline 20 & 3127 & -19901 & 68832 \\
\hline 21 & 786 & -19966 & 108744 \\
\hline 22 & -13939 & 37556 & 65229 \\
\hline 23 & -49052 & 223807 & -236055 \\
\hline 24 & -80276 & 508523 & -739289 \\
\hline 25 & 21450 & 321314 & 101404 \\
\hline 26 & 515846 & -2052462 & 7201383 \\
\hline 27 & 1411017 & -8417723 & 26255714 \\
\hline 28 & 1160761 & -13374892 & 43505098 \\
\hline 29 & -4793764 & 10841423 & -17552274 \\
\hline 30 & -20340586 & 112595914 & -291420026 \\
\hline 31 & -29699360 & 260687001 & -674637832 \\
\hline 32 & 33165914 & 70989018 & 27442 \\
\hline 33 & 256169433 & -1341964856 & 4426763291 \\
\hline 34 & 495347942 & -4108283969 & 12910062402 \\
\hline
\end{tabular}

Table 1. Large- $q$ series for the bulk, surface and corner free energies of the chromatic polynomial on the square lattice, in terms of the variable $z=1 /(q-1)$. We give the coefficients of $\exp \left(f_{\mathrm{b}}\right)=\frac{(q-1)^{2}}{q} \sum_{k=0}^{\infty} \alpha_{k} z^{k}, \exp \left(f_{\mathrm{s}}\right)=\sum_{k=0}^{\infty} \beta_{k} z^{k}$, and $\exp \left(f_{\mathrm{c}}\right)=\sum_{k=0}^{\infty} \gamma_{k} z^{k}$. 


\begin{tabular}{|c|c|c|c|}
\hline$k$ & $\alpha_{k}$ & $\beta_{k}$ & $\gamma_{k}$ \\
\hline 35 & -127736296 & -3304416038 & 9737827437 \\
\hline 36 & -3068121066 & 14960606999 & -49131891078 \\
\hline 37 & -7092358808 & 58237169596 & -184470253912 \\
\hline 38 & -1024264966 & 65268280922 & -183956055539 \\
\hline 39 & 35697720501 & -162368154719 & 621518352427 \\
\hline 40 & 91243390558 & -767619757924 & 2660084937207 \\
\hline 41 & 25789733672 & -975329692910 & 3075466954690 \\
\hline 42 & -420665229170 & 1872486336165 & -7500763944932 \\
\hline 43 & -1089052872105 & 9701425034093 & -34822638005931 \\
\hline 44 & -238516756366 & 12262136381593 & -38841312202313 \\
\hline 45 & 5101697398582 & -24192583755347 & 104412348649015 \\
\hline 46 & 12146149238921 & -118764516172484 & 448320847685638 \\
\hline 47 & -598931311074 & -130312353695974 & 421063156900936 \\
\hline 48 & -63338329084478 & 346985943489639 & -1507607631713074 \\
\hline 49 & -125863684143541 & 1412474002216034 & -5446631048235906 \\
\hline 50 & 71523371777335 & 1098760305055003 & -3234717862390686 \\
\hline 51 & 790320197578279 & -5183269098276812 & 22683201852542681 \\
\hline 52 & 1200257719380100 & -16304598291388925 & 64013086293590143 \\
\hline 53 & -1721302814683702 & -5448655170720498 & 8336294391279875 \\
\hline 54 & -9694394826555237 & 75813721738614619 & -329171853716322290 \\
\hline 55 & -10463394611604378 & 182868102163636571 & -718400221457685146 \\
\hline 56 & 29575960135787439 & -33129258404821172 & 310674267788777570 \\
\hline 57 & 115472492262862427 & -1051108217408277081 & 4563895897602278816 \\
\hline 58 & 85395764100885186 & -2017022924530473193 & 7953356311623456864 \\
\hline 59 & -421383549369627730 & 1373959176501915516 & -7763050320815504003 \\
\hline 60 & -1343046574902992458 & 13749175266084267367 & -60017851952733958335 \\
\hline 61 & -736061882807769534 & 22595026341744522020 & -90527220474610273313 \\
\hline 62 & 5291983916641725051 & -23294356475365888405 & 121713573010967365453 \\
\hline 63 & 15613586538385554242 & -172293246256852139137 & 768987768617382802911 \\
\hline 64 & 8244136015720127003 & -268117182162694379708 & 1123758858144026736112 \\
\hline 65 & -61540273845219665190 & 298116598787375322521 & -1553871017012870302628 \\
\hline 66 & -186576584391637858059 & 2127014580990734112356 & -9884276597620937431531 \\
\hline 67 & -119156912656052312977 & 3423100771562123299025 & -15168542715543012752367 \\
\hline 68 & 700439920487427255618 & -3325599173839179924758 & 18496951095018457216592 \\
\hline 69 & 2311567965527717105415 & -26543042275747617969190 & 130030721001519590513331 \\
\hline 70 & 1784944419294044372980 & -45519479688835787926364 & 210058368547617077899237 \\
\hline
\end{tabular}

Table 1. (continued.) 


\begin{tabular}{|r|r|r|r|}
\hline$k$ & $\alpha_{k}$ & $\beta_{k}$ & $\gamma_{k}$ \\
\hline 71 & -8190295787656754694494 & 36371250229750107503644 & -228752490963172501272677 \\
72 & -29120128759101310463081 & 336827820960866947369728 & -1729404450220843332781816 \\
73 & -24509224734165974718725 & 600304759572952426851130 & -2809877317417695436535077 \\
74 & 99609960301714320809469 & -429784892999554830063487 & 3052097912541295946004847 \\
75 & 363523561198034374612305 & -4289105251916055148255439 & 22691488586148969734635242 \\
76 & 307076608628563447831354 & -7658381117547376351931511 & 35816140480454963887649892 \\
77 & -1234243320099804373986722 & 5508060829297214833104965 & -41473851023699412644120745 \\
78 & -4471768398561919868797416 & 54201975484843398706779272 & -291274582715872945456529116 \\
79 & -3688741741221487636404873 & 95450369797304235548078036 & \\
\hline
\end{tabular}

Table 1. (continued.)

of the free-energy series, which is supposed to behave like $\sim\left(z-z_{\ell}\right)^{\lambda_{\ell}}$ as $z \rightarrow z_{\ell}$. Proceeding exactly as described in section 5.5.3 of [11], one obtains for the bulk free energy the first-order $(K=1)$ approximants

$$
\begin{array}{ll}
z_{1}=0.24(4) \pm 0.40(5) i & \lambda_{1}=-1.8(7) \\
z_{2}=0.64(7) & \lambda_{2}=-1.6(7) \\
z_{3}=-1.00(1) & \lambda_{3}=0.99(6)
\end{array}
$$

while the second-order $(K=2)$ approximants read

$$
\begin{array}{ll}
z_{1}=0.24(4) \pm 0.40(3) i & \lambda_{1}=-2.4(7) \\
z_{2}=0.62(10) & \lambda_{2}=-1.3(16)
\end{array}
$$

Somewhat disappointingly, these results hardly improve on those reported in [11, despite of the substantial extensions of the series. The same is true for the reanalysis of the surface and corner free energies.

\subsection{Triangular lattice series}

For the triangular lattice, we can use exactly the same finite-lattice weights as for the square lattice. Taking again $m_{\max }=20$ and $k=41$ corresponds to a maximum dimension of the transfer matrices of $M_{19}=18199284$. The error term in (23) $-(25)$ is now $O\left(z^{k}\right)$, and we know of no special tricks for obtaining an extra term. Therefore, we obtain in this case the three series to order $z^{40}$ included.

In a couple of important papers, Baxter [19, 6] has shown that the triangularlattice chromatic polynomial is an integrable system. In particular, he has set up the Bethe Ansatz equations and derived three different analytical expressions for the bulk free energy $f_{\mathrm{b}}$. The question which one of the three expressions dominates for a given real - or even complex - value of $q$ turns out to be very intricate [6], and has recently been the subject of a careful reanalysis [8]. The expression which is dominant for real 
$q \in(-\infty, 0] \cup[4, \infty)$ turns out to be

$$
\mathrm{e}^{f_{\mathrm{b}}}=-\frac{1}{x} \prod_{j=1}^{\infty} \frac{\left(1-x^{6 j-3}\right)\left(1-x^{6 j-2}\right)^{2}\left(1-x^{6 j-1}\right)}{\left(1-x^{6 j-5}\right)\left(1-x^{6 j-4}\right)\left(1-x^{6 j}\right)\left(1-x^{6 j+1}\right)}
$$

where the previous expansion variable (26) has now been traded for $x$ which is defined by

$$
q=2-x-\frac{1}{x}
$$

As expected, our series expansion validates (33) to order $x^{40}$ included.

The analytic computation of $f_{\mathrm{s}}$ and $f_{\mathrm{c}}$ was not attempted in [19, 6]. Presumably $f_{\mathrm{s}}$ could be derived by a substantial adaptation of Baxter's work in order to impose non-periodic boundary conditions in his transfer matrix formalism. On the other hand, the derivation of $f_{\mathrm{c}}$ appears to be a much harder problem, since this would require precise knowledge of the correspondence between boundary states in the Bethe setup and the initial lattice model, as well as the ability to compute scalar products in the Bethe formalism.

The series expansions that we obtain for $f_{\mathrm{s}}$ and $f_{\mathrm{c}}$ read as follows

$$
\begin{aligned}
\mathrm{e}^{f_{\mathrm{s}}}= & 1-2 x+x^{2}+2 x^{3}-2 x^{4}-x^{6}+2 x^{7}+2 x^{8}-4 x^{9}-2 x^{11}+7 x^{12}- \\
& 8 x^{14}+4 x^{15}-5 x^{16}+12 x^{17}-12 x^{19}+4 x^{20}-10 x^{21}+24 x^{22}- \\
& 2 x^{23}-19 x^{24}+2 x^{25}-15 x^{26}+46 x^{27}-10 x^{28}-24 x^{29}-x^{30}- \\
& 24 x^{31}+77 x^{32}-22 x^{33}-25 x^{34}-16 x^{35}-35 x^{36}+118 x^{37}- \\
& 41 x^{38}-14 x^{39}-45 x^{40}+O\left(x^{41}\right) \\
\mathrm{e}^{f_{\mathrm{c}}}= & 1+x-3 x^{3}-5 x^{4}-6 x^{5}+2 x^{6}+11 x^{7}+21 x^{8}+9 x^{9}-12 x^{10}- \\
& 41 x^{11}-33 x^{12}+72 x^{14}+82 x^{15}+40 x^{16}-102 x^{17}-169 x^{18}- \\
& 139 x^{19}+103 x^{20}+273 x^{21}+319 x^{22}-50 x^{23}-398 x^{24}-612 x^{25}- \\
& 111 x^{26}+517 x^{27}+1066 x^{28}+454 x^{29}-537 x^{30}-1686 x^{31}- \\
& 1067 x^{32}+398 x^{33}+2500 x^{34}+2079 x^{35}+76 x^{36}-3521 x^{37}- \\
& 3641 x^{38}-1138 x^{39}+4643 x^{40}+O\left(x^{41}\right) .
\end{aligned}
$$

It is seen that the coefficients are numerically much smaller than those for the square lattice (see Table 1). This in itself is not surprising, since the radius of convergence is 1 in the $x$ variable (triangular-lattice case), while that of the $z$ variable (square-lattice case) is $\simeq \frac{1}{2}$ (and presumably exactly $\frac{1}{2}$ in view of [26]). What is truly surprising is that the coefficients are integers! It thus looks reasonable to try to conjecture exact expressions.

In analogy with other problems where both bulk and surface properties have been worked out, one would expect the periodicity of 6 in the product formula (33) to be doubled to 12 in the corresponding surface quantities. Taking this as a clue suggests trying for $\mathrm{e}^{f_{\mathrm{s}}}$ and $\mathrm{e}^{f_{\mathrm{c}}}$ product formulae of the form

$$
\prod_{j=1}^{\infty} \prod_{k=0}^{11}\left(1-x^{12 j-k}\right)^{\alpha_{k}}
$$


for some exponents $\alpha_{k}$ to be determined. Knowledge of the coefficients of $x^{1}, \ldots, x^{12}$ from (35)-(36) allows us to determine $\alpha_{0}, \ldots, \alpha_{11}$; we can then test our conjectural formula against the coefficients of $x^{13}, \ldots, x^{40}$.

In this way we have obtained the conjecture

$$
\mathrm{e}^{f_{\mathrm{s}}}=\prod_{j=1}^{\infty}\left(\frac{\left(1-x^{12 j-11}\right)\left(1-x^{12 j-6}\right)\left(1-x^{12 j-5}\right)\left(1-x^{12 j-4}\right)}{\left(1-x^{12 j-9}\right)\left(1-x^{12 j-8}\right)\left(1-x^{12 j-7}\right)\left(1-x^{12 j-2}\right)}\right)^{2}
$$

for the surface free energy, and

$$
\begin{aligned}
\mathrm{e}^{f_{c}}=\prod_{j=1}^{\infty}\left(1-x^{12 j-11}\right)^{-1}\left(1-x^{12 j-10}\right)\left(1-x^{12 j-9}\right)^{3}\left(1-x^{12 j-8}\right)^{2} \times \\
\left(1-x^{12 j-7}\right)^{4}\left(1-x^{12 j-6}\right)^{-3}\left(1-x^{12 j-5}\right)\left(1-x^{12 j-4}\right)^{-3} \times \\
\quad\left(1-x^{12 j-3}\right)^{5}\left(1-x^{12 j-2}\right)^{2}\left(1-x^{12 j-1}\right)^{2}\left(1-x^{12 j}\right)
\end{aligned}
$$

for the corner free energy. The validation of (38)-(39) by $40-12=28$ independent checks means that both conjectures are established beyond any reasonable doubt.

\subsection{Limits $q \rightarrow 0$ and $q \rightarrow 4$}

It is obvious that the product formulae (33), (38) and (39) converge for $|x|<1$, since this is true for each individual factor in the product (by taking logarithms). It remains to discuss the limiting cases $x \rightarrow 1$ (i.e., $q \rightarrow 0$ ) and $x \rightarrow-1$ (i.e., $q \rightarrow 4$ ). The latter case -four-colouring the vertices of the triangular lattice - can be shown to be equivalent to other physically interesting problems, such as three-colouring the edges

of the hexagonal lattice [22, 23], or fully-packed loops on the hexagonal lattice with fugacity $n_{\text {loop }}=2$ [24].

In the $x \rightarrow 1$ case, we write $x=1-\epsilon$ for $\epsilon \ll 1$. Then, to leading order, we have for each factor in the products $1-x^{\alpha} \simeq \alpha \epsilon$. This leads to

$$
\begin{aligned}
\lim _{x \rightarrow 1} \mathrm{e}^{f_{\mathrm{b}}} & =-\prod_{j=1}^{\infty} \frac{(6 j-3)(6 j-2)^{2}(6 j-1)}{(6 j-5)(6 j-4)(6 j)(6 j+1)} \\
& =\frac{3^{3 / 2} \Gamma\left(\frac{1}{3}\right)^{9}}{(2 \pi)^{5} \simeq 3.770919 \cdots,} \\
\lim _{x \rightarrow 1} \mathrm{e}^{f_{\mathrm{s}}} & =\prod_{j=1}^{\infty}\left[\frac{(12 j-11)(12 j-6)(12 j-5)(12 j-4)}{(12 j-9)(12 j-8)(12 j-7)(12 j-2)}\right]^{2} \\
& =\frac{1}{2 \pi}\left[\frac{\Gamma\left(\frac{5}{12}\right) \Gamma\left(\frac{1}{4}\right)}{\Gamma\left(\frac{1}{6}\right)}\right]^{2} \simeq 0.305639 \cdots, \\
\lim _{x \rightarrow 1} \mathrm{e}^{f_{\mathrm{c}}} & =0 .
\end{aligned}
$$

In the $x \rightarrow-1$ case, we write $x=-1+\epsilon$ for $\epsilon \ll 1$. When $\alpha$ is even, we have then $1-x^{\alpha} \simeq \alpha \epsilon$ as before. And when $\alpha$ is odd, $1-x^{\alpha} \simeq 2$ to leading order. This leads to

$$
\lim _{x \rightarrow-1} \mathrm{e}^{f_{\mathrm{b}}}=\prod_{j=1}^{\infty} \frac{(3 j-1)^{2}}{(3 j-2)(3 j)}=\frac{3 \Gamma\left(\frac{1}{3}\right)^{3}}{4 \pi^{2}} \simeq 1.460998 \cdots,
$$




$$
\begin{aligned}
\lim _{x \rightarrow-1} \mathrm{e}^{f_{\mathrm{s}}} & =\prod_{j=1}^{\infty}\left[\frac{(6 j-3)(6 j-2)}{(6 j-4)(6 j-1)}\right]^{2}=4^{1 / 3} \simeq 1.587401 \cdots, \\
\lim _{x \rightarrow-1} \mathrm{e}^{f_{\mathrm{c}}} & =\infty
\end{aligned}
$$

It is remarkable that $f_{\mathrm{c}}$ diverges in both limits $x \rightarrow \pm 1$.

\section{Discussion}

In this paper we have used the finite-lattice method to obtain large- $q$ series for the bulk, surface and corner free energies-denoted $f_{\mathrm{b}}, f_{\mathrm{s}}$ and $f_{\mathrm{c}}$-for chromatic polynomials on regular two-dimensional lattices. Long series could be obtained due to a technical improvement of the transfer matrix method, which is specific to the antiferromagnetic zero-temperature limit of the Potts model, and amounts to obtaining a factorisation of $T$ in terms of sparse matrices, each of dimension $\sim 3^{m}$, where $m$ is the strip width. Although this applies in principle to any regular lattice, we have focussed here on the most commonly studied lattices (square and triangular).

For the square lattice the improvement allowed us to add 32 new terms to the existing series [11. Unfortunately, the reanalysis of the singularities of this series, using the method of differential approximants, did not lead to any significant improvement over [11]. The focus in this paper was therefore rather on the triangular lattice, where the series permitted us to conjecture the exact analytical formulae (38)-(39) for $f_{\mathrm{s}}$ and $f_{\mathrm{c}}$, and to confirm the correctness of Baxter's [19, 6] result (33) for $f_{\mathrm{b}}$.

It should be noticed that going to $m_{\max }=20$ leads to 79 terms in the square-lattice series, but only to 40 terms for the triangular lattice. Longer series could be obtained in the latter case by using a version of the finite-lattice method which is specifically adapted to the triangular lattice [25]. However, since our series have enabled us to conjecture the exact (infinite-order) results (38)-(39) the motivation for extracting further terms of the series is admittedly rather scarce. Indeed, the series reported here already provide $40-12=28$ independent checks of the proposed expressions (38)-(39).

It would be interesting to elucidate the physical mechanism by which $f_{\mathrm{c}}$ diverges in the limits $q \rightarrow 0$ and $q \rightarrow 4$.

It is regrettable that the series obtained here do not provide any information about

the regions where the chromatic polynomial is a critical system - that is, $q \in[0,3]$ for the square lattice [26, 13, 14, 3, 7, 10] and $q \in[0,4]$ for the triangular lattice [19, 6, 8, 10]. The reason is that $q=\infty$ seems to be the only viable expansion point for the finitelattice method.

It is possible that the fact that non-nearest neighbour constraint can be made local conceals some deeper representation theoretical meaning. It is well-known that the generic representation theory of the Temperley-Lieb algebra, which is central to the algebraic formulation of the planar Potts model, undergoes profound modifications when $q=4 \cos (\pi /(p+1))^{2}$ with $p$ integer (or rational) [27]. It might be that similar modifications occur upon taking the particular value $v=-1$ for the temperature 
variable. It should be noticed that some steps toward an algebraic formulation of the chromatic polynomial have been taken in [28].

\section{Acknowledgements}

This work was supported by the Agence Nationale de la Recherche (grant ANR-06BLAN-0124-03). The author expresses his gratitude to J. Salas and A.D. Sokal for a careful reading of the manuscript; and in particular to J. Salas for reanalysing the square-lattice series along the lines of [11].

\section{References}

[1] G.D. Birkhoff, Ann. Math. 14, 42 (1912).

[2] H. Whitney, Bull. Amer. Math. Soc. 38, 572 (1932).

[3] J. Salas and A.D. Sokal, J. Stat. Phys. 104, 609 (2001) arXiv:cond-mat/0004330.

[4] K. Appel and W. Haken, Bull. Amer. Math. Soc. 82, 711 (1976).

[5] G.D. Birkhoff and D.C. Lewis, Trans. Amer. Math. Soc. 60, 355 (1946).

[6] R.J. Baxter, J. Phys. A 20, 5241 (1987).

[7] J.L. Jacobsen and J. Salas, J. Stat. Phys. 104, 701 (2001) arXiv:cond-mat/0011456.

[8] J.L. Jacobsen, J. Salas and A.D. Sokal, J. Stat. Phys. 112, 921 (2003) arXiv:cond-mat/0204587.

[9] J.L. Jacobsen and J. Salas, J. Stat. Phys. 122, 705 (2006) arXiv:cond-mat/0407444].

[10] J.L. Jacobsen and J. Salas, Nucl. Phys. B 783, 238 (2007) arXiv:cond-mat/0703228.

[11] J. Salas and A.D. Sokal, J. Stat. Phys. 135, 279 (2009) arXiv:0711.1738.

[12] J. Salas and A.D. Sokal, arXiv:1002.3761.

[13] H. Saleur, Comm. Math. Phys. 132, 657 (1990).

[14] H. Saleur, Nucl. Phys. B 360, 219 (1991).

[15] G.F. Royle, Annals Combin. 12, 195 (2008) arXiv:math/0511304.

[16] H.W.J. Blöte and M.P. Nightingale, Physica A 112, 405 (1982).

[17] T. de Neef and I.G. Enting, J. Phys. A 10, 801 (1977).

[18] A.V. Bakaev and V.I. Kabanovich, J. Phys. A 27, 6731 (1994).

[19] R.J. Baxter, J. Phys. A 19, 2821 (1986).

[20] C.M. Fortuin and P.W. Kasteleyn, Physica 57, 536 (1972).

[21] I.G. Enting, J. Phys. A 11, 563 (1978).

[22] R.J. Baxter, J. Math. Phys. 11, 784 (1970).

[23] C. Moore and M.E.J. Newman, J. Stat. Phys. 99, 629 (2000) arXiv:cond-mat/9902295.

[24] J. Kondev and C.L. Henley, Phys. Rev. Lett. 73, 2786 (1994).

[25] I.G. Enting, J. Phys. A 20, 1485 (1987).

[26] R.J. Baxter, Proc. Roy. Soc. London A 383, 43 (1982).

[27] V. Pasquier and H. Saleur, Nucl. Phys. B 330, 523 (1990).

[28] P. Fendley and V. Krushkal, Geometry and Topology 13, 709 (2009) arXiv:0711.0016. 